\title{
Percutaneous electrochemotherapy in primary and secondary liver malignancies - local tumor control and impact on overall survival
}

\author{
Hannah Spallek ${ }^{1}$, Peter Bischoff ${ }^{1}$, Willi Zhou ${ }^{1}$, Francesca de Terlizzi², Fabian Jakob³ \\ Attila Kovàcs ${ }^{1}$ \\ ${ }^{1}$ Clinic for Diagnostic and Interventional Radiology and Neuroradiology, MediClin Robert Janker Klinik, Bonn, Germany
${ }^{2}$ IGEA Clinical Biophysics, Laboratory Carpy, Modena, Italy
${ }^{3}$ Clinic for Radiology and Nuclear Medicine, University Hospital Schleswig Holstein, Campus Luebeck, Luebeck, Germany
}

Radiol Oncol 2022; 56(1): 102-110.

Received 19112021

Accepted 24122021

Correspondence to: Attila Kovàcs, Clinic for Diagnostic and Interventional Radiology and Neuroradiology, MediClin Robert Janker Klinik, Bonn, Germany. E-mail: Attila.Kovacs@mediclin.de

Disclosure: No potential conflicts of interest were disclosed.

This is an open access article under the CC BY-NC-ND license (http://creativecommons.org/licenses/by-nc-nd/4.0/).

Background. Local nonsurgical tumor ablation currently represents a further option for the treatment of patients with liver tumors or metastases. Electrochemotherapy (ECT) is a welcome addition to the portfolio of local therapies. A retrospective analysis of patients with liver tumors or metastases treated with ECT is reported. Attention is given to the safety and efficacy of the treatment over time.

Patients and methods. Eighteen consecutive patients were recruited with measurable liver tumors of different histopatologic origins, mainly colorectal cancer, breast cancer, and hepatocellular cancer. They were treated with percutaneous ECT following the standard operating procedures (SOP) for ECT under general anaesthesia and muscle relaxation. Treatment planning was performed based on MRI preoperative images. The follow-up assessment included contrast-enhanced MR within at least 1-3 months after treatment and then after 5, 7, 9, 12, and 18 months until progression of the disease or death.

Results. Only mild or moderate side effects were observed after ECT. The objective response rate was $85.7 \%$ (complete response $61.9 \%$, partial 23.8\%), the mean progression-free survival (PFS) was $9.0 \pm 8.2$ months, and the overall survival (OS) was $11.3 \pm 8.6$ months. ECT performed best (PFS and OS) in lesions within 3 and $6 \mathrm{~cm}$ diameters ( $p=0.0242$, $p=0.0297)$. The effectiveness of ECT was independent of the localization of the lesions: distant, close or adjacent to vital structures. Progression-free survival and overall survival were independent of the primary histology considered.

Conclusions. Electrochemotherapy provides an effective valuable option for the treatment of unresectable liver metastases not amenable to other ablative techniques.

Key words: electrochemotherapy; liver metastases

\section{Introduction}

Globally, liver cancer ranks sixth for cancer incidence and fourth for cancer deaths, being the second leading cause of cancer-related years of life lost. During the next decade, a further increase in the number of new cases of primary liver cancer is predicted each year in most countries as a result of changes in risk factors. ${ }^{1,2}$

Globally, colon and rectal cancer ranks third for cancer incidence and second for cancer deaths. ${ }^{1}$ Population-based studies have shown that $25-30 \%$ of patients diagnosed with colorectal cancer (CRC) develop liver metastases during the course of their 
disease. Indications for curative-intended treatment of CRC liver metastases have expanded in recent years. Unfortunately, despite oncological and surgical advances, only $25 \%$ of patients affected are amenable to resection. ${ }^{3,4}$

Local nonsurgical interventional tumor ablation currently represents a further option for the treatment of cancer patients. Local treatments can be divided into thermal (radiofrequency or microwave ablation and cryoablation) and nonthermal treatments (high precision radiotherapy, brachytherapy and electroporation).

The European Society of Medical Oncology (ESMO) included local ablation procedures in the current consensus guidelines on the treatment of metastatic colorectal cancer (mCRC). ${ }^{5}$

The choice of therapy is determined by the number, size, configuration and location or environment of the target lesion. Thermal ablation techniques are emerging as alternative treatment options to open surgery for both primary and secondary hepatic tumors. ${ }^{6} \mathrm{~A}$ disadvantage of RFA is the therapeutic limitation to smaller target lesions up to $3.5 \mathrm{~cm}$ in diameter; in the case of microwave and cryoablation, the ablation zone can be enlarged up to $5 \mathrm{~cm}$ in tumor diameter. However, hyperthermia-based technologies have some limitations, including heat sink effects in the proximity of large blood vessels, the risk of causing cholestasis when treating lesions close to the thermosensitive bile ducts or damaging critical structures if proximal to the hepatic portal Glisson's capsule or diaphragm, or if located on the intra-abdominal free surface.

A special form of local nonthermal ablation is internal radiation (interstitial brachytherapy), which uses radiation with a very limited range because it is limited in the proximity of organs vulnerable to radiation; it cannot be repeated, and furthermore, some tumor entities are not radiosensitive. In such cases, chemoablation, such as electrochemotherapy (ECT), is a welcome addition to the portfolio of local therapies.

ECT is a local ablative technique that utilizes electroporation for enhanced drug (bleomycin or cisplatin) delivery to cells by generating transient permeation structures in the cell membrane. ${ }^{7,8}$ Over the past 20 years, ECT has been shown to have proven effectiveness in the treatment of cutaneous, subcutaneous, mucosal, or deep-seated tumors of various histologies and in different body sites. ${ }^{9-11}$

The international, multicenter clinical study European Standard Operating Procedures for Electrochemotherapy (ESOPE) developed the Standard Operating Procedures for ECT on cutane- ous tumors with the Cliniporator ${ }^{\mathrm{TM}}$ Device (IGEA S.p.A., Carpi, Italy). ${ }^{8}$ Based on its effectiveness for cutaneous tumors, ECT is now being developed and has been shown to be feasible, safe, and effective for deep-seated tumors, such as liver tumors. ECT can be used near collagenous structures such as vessels and bile ducts ${ }^{12}$, and it is repeatable and suitable as a local therapy between chemotherapy cycles. ECT has the potential to close relevant gaps in the spectrum of local ablative therapies, enabling the treatment of i) lesions that are too large for thermal ablation, ii) nonradiation-sensitive tumors or iii) lesions located in the immediate vicinity of radiation- or temperature-vulnerable organs. ECT is specifically suitable for the treatment of liver metastases located centrally, close to the capsule or in proximity of the major vessels, which are not resectable and not suitable for radiofrequency ablation or microwave ablation due to the heat sink effect. The safety of ECT in the treatment of metastases located near large liver vessels was also proven in animal models. ${ }^{13,14}$ In cancer patients, ECT is well tolerated, with few side effects and no relevant pain, nausea or systemic side effects. ${ }^{15-18}$

In this study, we present a retrospective analysis of patients with liver tumors or metastases treated with ECT at our institution. This is the first realworld clinical experience on percutaneous application of ECT in the liver in a large cohort of patients. Attention is given to the safety and efficacy of the treatment over time.

\section{Patients and methods}

In this cohort study, 18 patients with measurable liver tumors of different histopathologic origins were recruited: colorectal cancer, breast cancer, hepatocellular cancer, ovarian cancer, anal cancer, non-small cell lung cancer (NSCLC) and cancer of unknown primary origin (CUP). They were treated with ECT between June 2018 and June 2020. The study was conducted according to the Helsinki declaration. The patients signed an informed consent form. The study was approved by the Committee for Medical Ethics of the Institution.

\section{Imaging}

Standard pretreatment evaluation of patients with liver tumors included liver MRI with a hepatospecific contrast agent and CT of the thorax and abdomen, including the pelvis at least 1 month before ECT. MRI was performed using a GE Signa Hdxt 


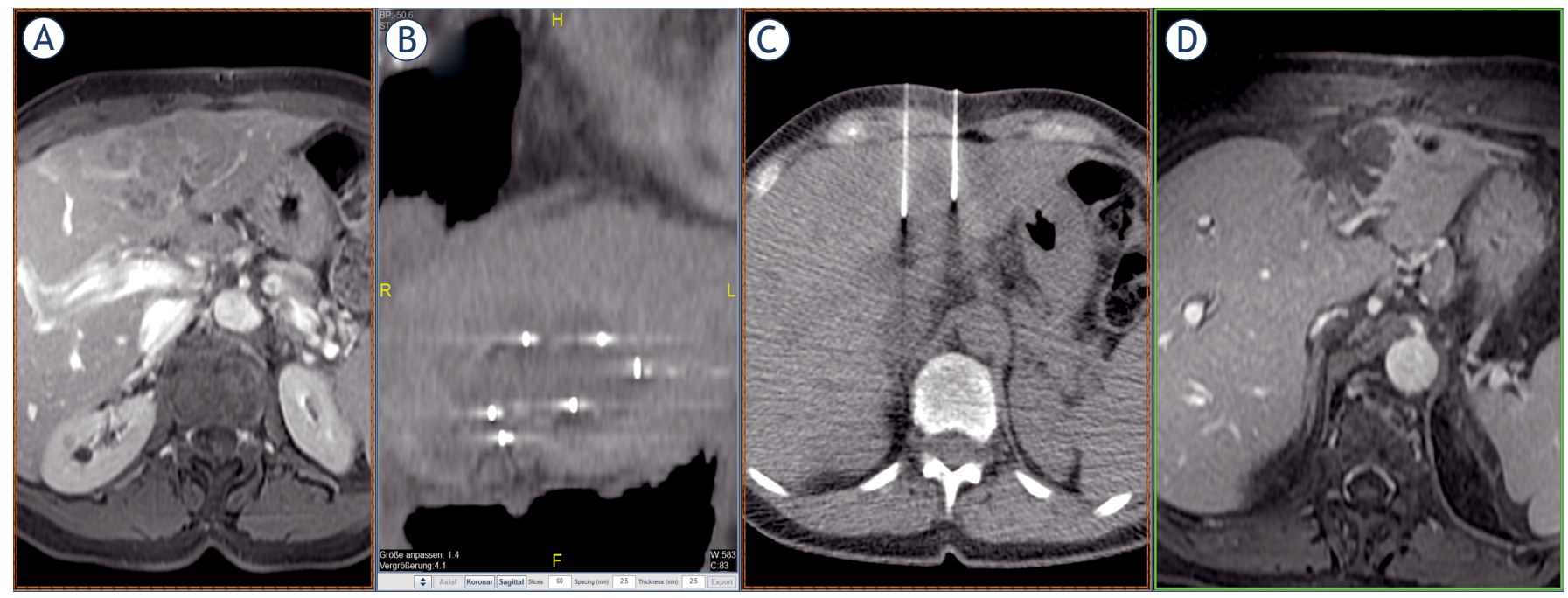

FIGURE 1. (A) Solitary liver metastasis from a breast carcinoma in a challenging location between the left and right lobes of the liver, not amenable to surgical resection and progressive under various lines of systemic chemotherapy. The dimensions of the metastasis in segment IVa/b adjacent to segment VIII were $4 \times 7 \times 5 \mathrm{~cm}$ (volume $70 \mathrm{cc}$ ). (B) Position of the electrodes in the coronary reconstruction. The aim is to achieve the most uniform coverage of the target lesion by the electrodes. (C) Position of the electrodes in axial cross-sectional imaging. This image shows another essential requirement for the therapeutic success of ECT - the parallelism of the electrodes. (D) The most recent imaging control, complete two years after the ECT procedure, shows complete chemoablation of the entire metastasis, thus formally complete remission of the target lesion without residual or marginal recurrence.

1.5T using standard imaging sequences for the liver: tra T2w fs, tra DWI, tra T1w nat, tra and cor ce T1w. The patients were reviewed by a multidisciplinary team. The follow-up assessment included contrast-enhanced MRI of the liver within at least 1-3 months after treatment and then after 5, 7, 9, 12, 18 months and/or until progression of the disease or death.

\section{Electrochemotherapy}

Freehand electrodes are used for the percutaneous therapy of parenchymatous organs or for intraoperative positioning. Depending on the size, configuration and localization of the target region, different active electrode tips, shaft lengths and thicknesses can be selected. A maximum of 6 probes can be operated synchronously. Therapy planning is software-based: the parallel positioning of the probes at a defined distance from each other plays an essential role in the optimal therapeutic coverage of the target lesion. The aim is to achieve a distance of 2.0 to $2.5 \mathrm{~cm}$ from each other and a voltage to be applied of approx. 1000 volts per $\mathrm{cm}$. Treatment planning was performed based on MRI preoperative images. During ECT treatment, needle electrodes were percutaneously inserted based on the CT-fluoro guided planning images, and a distance of at least 0.5 and maximally $3.0 \mathrm{~cm}$ be- tween the electrodes was ensured. Electrodes were freely positionable single needle probes with a diameter of $1.2 \mathrm{~mm}$; a minimum of 2 and a maximum of 8 electrodes (median 6) were used per treatment (Table 2). In particular: in 8 patients electrodes 16 $\mathrm{cm}$ long and with an active part of $4 \mathrm{~cm}$, in 6 patients electrodes $16 \mathrm{~cm}$ long with an active part of $3 \mathrm{~cm}$, in 4 patients electrodes $20 \mathrm{~cm}$ long with $3 \mathrm{~cm}$ active part and in 1 patient electrodes $20 \mathrm{~cm}$ long with active part of $4 \mathrm{~cm}$ (IGEA, Carpi, Italy). The direction of electrode access was determined by the performing surgeon.

Since the pulses are delivered, among pairs of electrodes, the computation of the voltage to be applied was performed by the device for each pair of electrodes separately, and the appropriate electric field for each pair combined assured the complete coverage of a tumor. By sequentially activating the electrodes in this way, a larger tumor volume can be covered with sufficiently strong electric fields. ${ }^{19}$ The goal was to ensure $100 \%$ coverage of the clinical target volume with an electric field above 400 $\mathrm{V} / \mathrm{cm}$ and to limit the maximum current delivered to the tissue to be below $50 \mathrm{~A}$ (hardware limit of the IGEA Cliniporator Vitae pulse generator). ${ }^{20}$

ECT was performed using the same treatment protocol as defined by the SOP for ECT of cutaneous tumors regarding the drug dosage and electrical parameters (i.e., pulse duration and number of 
pulses) of electroporation. ${ }^{8,21}$ The needle electrodes were percutaneously inserted into the lesions under CT-fluoro guidance. The electrodes were connected to an electric pulse generator (Cliniporator VITAE, IGEA SpA, Carpi, Italy). Thereafter, the patients were given $15,000 \mathrm{U} / \mathrm{m}^{2}$ bleomycin intravenously in bolus. Eight electric pulses of $100 \mu \mathrm{s}$ duration were delivered between pairs of electrodes $8 \mathrm{~min}$ after the bleomycin injection, when the maximal pharmacological peak of bleomycin in the tumors was expected. Complete coverage of the tumor by repositioning of the electrodes should possibly be completed by 40 minutes thereafter. Care is also taken to ensure that the electrical pulses are delivered only in the refractory phase of the heart by automatic ECG synchronization to avoid interferences with the heart rhythm. ECT treatment takes place under general anaesthesia and muscle relaxation as a minimally invasive, usually percutaneous, procedure (Figure 1).

\section{Statistical analysis}

Descriptive statistics are reported as the mean, standard deviation, median and range for continuous variables and absolute numbers and percentage for categorical variables. Comparisons between groups were performed by ANOVA (continuous variables) and contingency tables and chi square tests (for categorical variables). A P value lower than 0.05 was considered statistically significant. Statistical analysis was performed with NCSS 9 (NCSS 9 Statistical Software (2013)). NCSS, LLC. Kaysville, Utah, USA, ncss.com/software/ncss).

\section{Results}

Eighteen patients were treated with ECT in the period June 2018 - June 2020 and were followed for a median time of 9 months (mean $11.3 \pm 8.6$ months). One patient was lost to follow-up. Three patients were treated with 2 lesions, all the others in a single lesion of the liver.

The characteristics of the cohort of patients are reported in Table 1 . The mean age was $64.3 \pm 11.1$ years (median 64, range 41-83 years).

Lesion sizes were $5.9 \pm 2.5 \mathrm{~cm}$ in the LA (long axis) direction (median 4.6, range 1.5-11.2 cm) and $5.4 \pm 2.1 \mathrm{~cm}$ in the SA (short axis) direction (median 5.5 , range $1.5-10 \mathrm{~cm}$ ). The overall mean volume was $129.6 \pm 137.3 \mathrm{~cm}^{3}$ (median 57, range 23-475 $\mathrm{cm}^{3}$ ). Table 2 shows other characteristics of the lesions and the technical parameters of ECT treatment.
TABLE 1. Demographic

\begin{tabular}{|c|c|c|}
\hline & $\mathbf{N}$ & $\%$ \\
\hline PATIENTS & 18 & \\
\hline \multicolumn{3}{|l|}{ GENDER } \\
\hline M & 8 & $44.4 \%$ \\
\hline $\mathrm{F}$ & 10 & $55.6 \%$ \\
\hline \multicolumn{3}{|l|}{ DIAGNOSIS } \\
\hline Colorectal cancer & 7 & $38.9 \%$ \\
\hline Breast cancer & 4 & $22.2 \%$ \\
\hline Hepatocellular cancer & 2 & $11.1 \%$ \\
\hline Ovarian cancer & 2 & $11.1 \%$ \\
\hline Anal cancer & 1 & $5.6 \%$ \\
\hline Cancer of unknown primary origin (CUP) & 1 & $5.6 \%$ \\
\hline Non-small cell lung cancer (NSCLC) & 1 & $5.6 \%$ \\
\hline TUMOURS TREATED & 21 & \\
\hline \multicolumn{3}{|l|}{ LIVER METASTASES } \\
\hline Synchronous & 8 & $44.4 \%$ \\
\hline Metachronous & 8 & $44.4 \%$ \\
\hline No & 2 & $11.2 \%$ \\
\hline \multicolumn{3}{|l|}{ METASTASES LOCATION } \\
\hline Liver only & 7 & $43.7 \%$ \\
\hline Liver + lung & 3 & $21.5 \%$ \\
\hline Liver + bone & 1 & $6.2 \%$ \\
\hline Liver + kidney & 1 & $6.2 \%$ \\
\hline Liver + lung + bone + brain & 1 & $6.2 \%$ \\
\hline Liver + bone + peritoneum & 1 & $6.2 \%$ \\
\hline Liver + pleural + bone & 1 & $6.2 \%$ \\
\hline Liver + retroperitoneal & 1 & $6.2 \%$ \\
\hline \multicolumn{3}{|l|}{ PREVIOUS TREATMENTS } \\
\hline Systemic therapy & 16 & $88.8 \%$ \\
\hline Liver surgery & 4 & $22.2 \%$ \\
\hline TACE & 8 & $44.4 \%$ \\
\hline TACE + RFA & 1 & $5.6 \%$ \\
\hline$T A C E+C P$ & 1 & $5.6 \%$ \\
\hline CRYOTH & 1 & $5.6 \%$ \\
\hline NO & 7 & $38.9 \%$ \\
\hline \multicolumn{3}{|l|}{ COMORBIDITIES* } \\
\hline Cardiac diseases & 6 & $33.3 \%$ \\
\hline Pulmonary diseases & 3 & $16.7 \%$ \\
\hline Liver diseases & 9 & $50.0 \%$ \\
\hline
\end{tabular}

* Cardiac diseases were cardiomyopathies, status post coronary bypass, status post aortocoronary venous bypass operation, valvular disease, pericardial effusion; pulmonary diseases were chronic obstructive pulmonary diseases; liver diseases were hematomas, ascites, cholestasis, hemochromatosis

$\mathrm{CP}=$ chemoperfusion; $\mathrm{CRYOTH}=$ cryotherapy; $\mathrm{F}=$ female; $\mathrm{M}=$ male; $\mathrm{N}=$ number; $\mathrm{RFA}=$ radiofrequency ablation; $\mathrm{TACE}=$ hepatic artery chemoembolization

\section{Safety/toxicity}

Only mild or moderate side effects were observed after ECT: in 16 of 21 patients, temporary (1st day) mild pain at the treated site; in 1 patient, CRP (C-reactive protein) elevation and leucocytosis were successfully treated with i.v. antibiotics; and in 1 patient, moderate pain due to a liver capsular hematoma, w.o. hemoglobin drop, successfully treated with ibuprofen/pantoprazole self-resolving after 10 days. 
TABLE 2. Lesions and treatment description

\begin{tabular}{lcc}
\hline & N & $\%$ \\
\hline LESIONS & 21 & $100 \%$ \\
\hline PLANNING MRI & 21 & $100 \%$ \\
\hline TYPE & & \\
$\quad$ Hypervascular & 2 & $9.5 \%$ \\
Intermediate & 14 & $71.4 \%$ \\
Hypovascular & 5 & $19.0 \%$ \\
CHALLENGING LOCATION* & & \\
Yes & 19 & $90.5 \%$ \\
No & 2 & $9.5 \%$ \\
VESSELS OR BILE DUCTS SURROUNDING THE METASTASES & & \\
Distant (> 10 mm) & 4 & $19.0 \%$ \\
Close (1 mm to 10 mm) & 6 & $28.6 \%$ \\
Adjacent (< 1 mm) & 11 & $52.4 \%$ \\
PREVIOUS LOCAL TREATMENT ON THE LESION & & \\
LOCal ablative therapy (LAT) & 0 & $0 \%$ \\
Transarterial Chemoembolization (TACE) & 6 & $28.6 \%$ \\
Chemoperfusion (CP) & 1 & $4.8 \%$ \\
Treatment-naive & 14 & $66.7 \%$ \\
\hline TECHNICAL SUCCESS & & \\
Yes & 20 & $95.2 \%$ \\
No & 1 & $4.8 \%$ \\
\# ELECTRODES PER TREATMENT & & \\
2 & & $4.8 \%$ \\
3 & 1 & $9.5 \%$ \\
4 & 2 & $4.8 \%$ \\
6 & 1 & $76.2 \%$ \\
8 & $4.8 \%$ \\
\hline
\end{tabular}

* Challenging location represented in liver were liver dome, vicinity of portal vein main trunk, vicinity of main bile duct

\section{Response to treatment}

The response to treatment was evaluated between 1 and 3 months after the ECT session; the overall response is reported in Table 3. Objective response rate was $85.7 \%$.

Mean progression-free survival (PFS) was 9.0 \pm 8.2 months. Three patients progressed during follow-up after 3, 5, and 7 months. The first underwent TACE and was in complete response (CR) after 6 months; the second underwent interstitial brachytherapy and was in CR after 2 months; the last underwent TACE + CRYO and was in CR after 11 months.

Mean overall survival (OS) was $11.3 \pm 8.6$ months. Three patients died for reasons related to liver metastases (14.3\%), 11 patients $(52.4 \%)$ died for other reasons, and 7 (33.3\%) were still alive.

Furthermore, response to treatment, PFS and OS were evaluated according to lesion size, histology of the primary tumor and location of the liver lesions. The results are shown in Table 4.

\section{Discussion}

Interventional oncology is the fastest developing area of interventional radiology. Minimally invasive, image-guided procedures are playing an increasingly important role in multimodal cancer therapy. $22-24$

In this study, we evaluated the effect of percutaneous ECT on liver tumors of different pathologic origins. Percutaneous ECT treatments of 21 lesions in 18 patients were included in the analysis. The lesions were close to the capsule or in proximity of the major vessels, not suitable for radiofrequency or microwave ablation due to the heat sink effect, and not surgically resectable. In $90.5 \%$ of cases, the lesions were in a challenging location (liver dome, near portal vein main trunk, near main bile duct).

The mean volume of treated lesions was $129.59 \pm$ $137.31 \mathrm{~cm}^{3}$, which is definitely larger than the volumes usually accessible for other minimally invasive procedures.

The most important advantage of minimally invasive technologies is that, in combination with standard therapies, they significantly increase overall survival compared to standard treatment alone. This has been proven in 2 different studies: the $\mathrm{CLOCC}^{22}$ and the SABR-COMET ${ }^{25}$ trials. In the CLOCC trial, patients with nonresectable colorectal cancer liver metastases were randomized either to receive systemic chemotherapy or a combination of systemic and minimally invasive therapies; the overall survival at 8 years was significantly improved in the combined arm versus the standard therapies arm ( $36 \%$ vs. $8 \%)$. In the same way, the SABR-COMET study analyzed the impact of stereotactic ablative radiotherapy (SABR) in combination with standard of care in the treatment of different oligometastatic patients from various cancers (breast, lung, colorectal, prostate) in comparison with standard of care alone. The first arm showed a superior 5-year survival (42.3\% vs. $17.7 \%)$. Other advantages of minimally invasive technologies are good tolerability, less impact on quality of life, fewer systemic side effects and tissue preservation when compared to classical surgery. Furthermore, except for radiotherapy, minimally invasive technologies are also repeatable.

Thermo and radioablative techniques are limited in some situations, for example, when target lesions have a size that exceeds the safe ablation zone of thermal procedures, usually estimated at $3.5 \mathrm{~cm}$ in diameter. ${ }^{26}$ On the other hand, radioablation is limited near radiation-sensitive organs. In these cases, ECT can truly be a valuable option, as it is 
TABLE 3. Response of target lesions evaluated between 1 and 3 months

\begin{tabular}{lcc}
\hline RESPONSE & N & $\%$ \\
\hline Complete response (CR) & 13 & $61.9 \%$ \\
\hline Partial response (PR) & 5 & $23.8 \%$ \\
\hline Stable disease (SD) & 1 & $4.8 \%$ \\
\hline Progressive disease (PD) & 0 & $0 \%$ \\
\hline Lost to follow-up & 2 & $9.5 \%$ \\
\hline
\end{tabular}

a combined tumor therapy that enhances the local effect of a systemically administered chemotherapeutic drug by reversible electroporation. ${ }^{26}$ The most commonly used chemotherapeutic agent is bleomycin, which, when combined with electroporation, has the major advantage of being cytotoxic regardless of the tumor's histology.

The aim of minimally invasive, local ablative procedures is to destroy primary and secondary malignancies efficiently and gently at the same time using image guidance. These novel techniques can be used for diverse applications ranging from curative intent for small localized tumors, down staging of large tumors for resection, or locoregional control and palliation of advanced disease. The choice of therapy is determined by the parameters "number, size and location" of the target lesions. Given that all standard local ablative procedures were available at the hospital that conducted the present study, a proactive decision was made to perform ECT in each individual case. Due to the aforementioned limitations of each procedure, it was most often the size, location and immediate environment of the target lesions that contraindicated thermal ablation or compromised its efficacy in the first place. In most decisions, from the therapist's point of view, ECT seemed to us to be the only alternative.

This technique has already been shown to be effective on cutaneous, subcutaneous and mucosal lesions ${ }^{10,11}$, with a response rate of $70-80 \%$, and to be particularly effective on basal cell carcinoma, with a complete remission of treated lesions up to $91 \%{ }^{11}$

ECT has also shown convincing results with deep-seated tumors. In a prospective multicenter study $^{27}$, Campanacci et al. evaluated ECT in the treatment of symptomatic bone metastases. The results on 102 patients from 11 European centers demonstrated that ECT is a safe and effective treatment for painful bone metastases resistant to other local treatments. Furthermore, a significant decrease in pain intensity and significantly better quality of life were observed after the ECT session and at later follow-up.

Several pilot studies on intraoperative ECT on liver metastases ${ }^{16,28-30}$, hepatocellular carcinoma ${ }^{31,32}$, perihilar cholangiocarcinoma ${ }^{33,34}$, vulvar cancer ${ }^{35-37}$, and renal cancer ${ }^{38}$ are available in the literature.

In a recently published phase II study on ECT in the treatment of colorectal liver metastases, the objective response rate (OR) per lesion was 75\%, with $63 \%$ complete response (CR) and $12 \%$ partial response (PR), while OR, CR and PR per patient were $59 \%, 44 \%$ and $15 \%$, respectively. The median response time was 20.8 months for metastases in CR and 9.8 months for metastases in PR. There was no difference in treatment response with regard to the location of the metastases, e.g., metastases in the central vs. peripheral location. The median overall survival of patients after ECT was 29.0 months. ${ }^{16}$ The histopathological assessment of some of these colorectal liver metastases after ECT treatment showed that most vessels $(>5 \mathrm{~mm})$ and biliary structures remained intact, while smaller blood vessels were damaged. This study shows that ECT can be safely applied to treat metastases in the immediate vicinity of the large blood vessels in the liver. ${ }^{12}$

Regarding hepatocellular carcinoma (HCC), a prospective phase II study was recently published on 24 patients with $32 \mathrm{HCC}$ lesions not suitable for other curative treatments according to the BCLC classification or refractory to previous surgery and different local ablative techniques. In this study, the treatment was proven to be equally effective for tumors located centrally and peripherally, with a median response rate per patient of $95.8 \%$ (79.2\% $\mathrm{CR}$ and $16.6 \% \mathrm{PR})$. The overall survival over 5 years of observation was $72.0 \% .^{31}$

In these studies, ECT treatment was performed intraoperatively as part of an open procedure. To the best of our knowledge, the present study is the largest case series about ECT on liver tumors with a percutaneous approach.

To date, only a few studies with a percutaneous approach to liver tumors using ECT have been published. ${ }^{15}$ Tarantino et al. 2017 demonstrated the efficiency and effectiveness of ECT in the treatment of six patients with portal vein thrombosis at the hepatic hilum, resulting in two patients with regained complete patency of the portal vein and three patients with a persistent avascular nontumoral shrinked thrombus; none of these patients developed a local recurrence. ${ }^{39}$ The same group 
TABLE 4. Response to treatment, progression-free survival and overall survival according to different subgroups of analysis

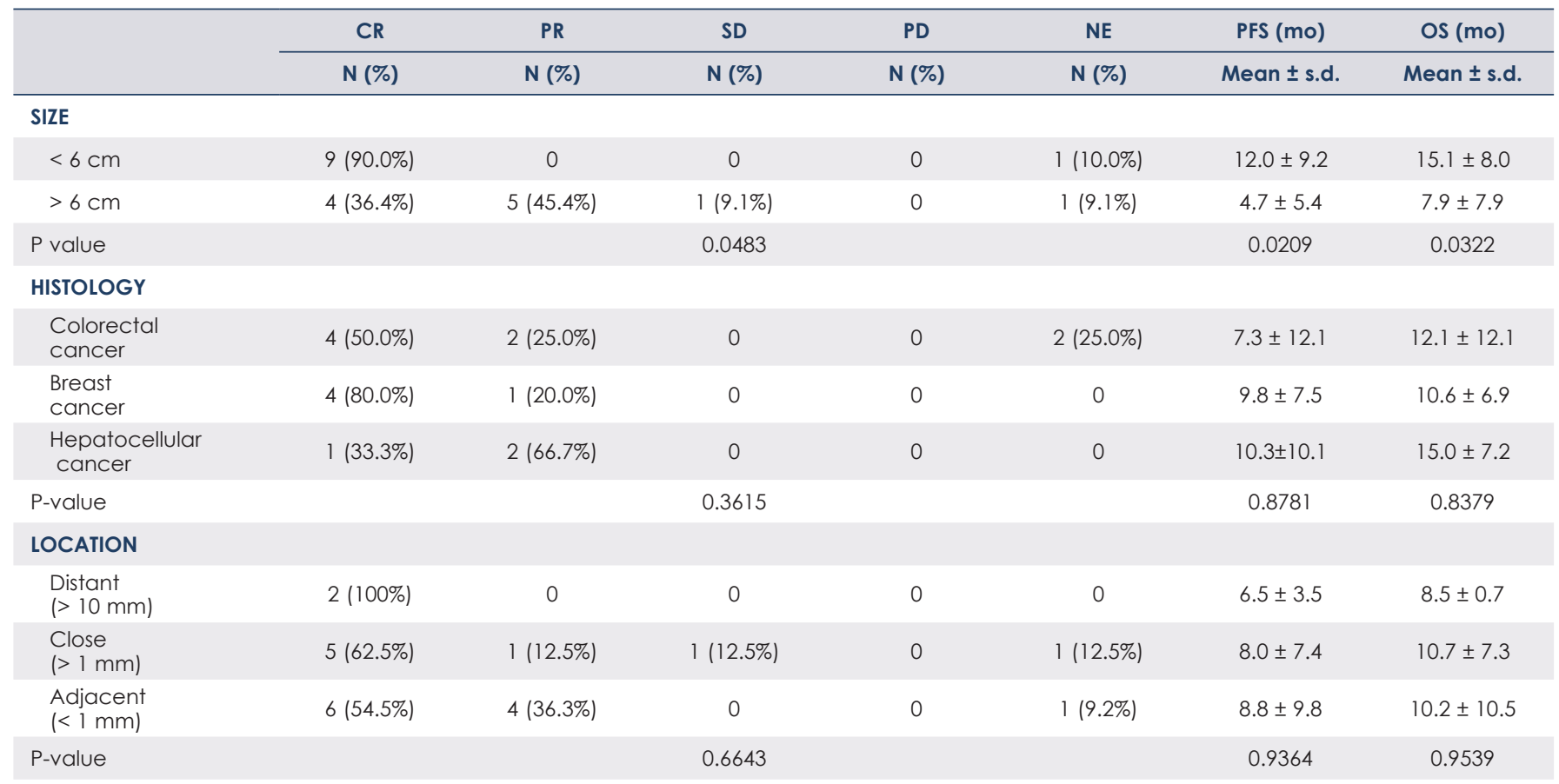

$\mathrm{CR}=$ comlete response; $\mathrm{NE}=$ no evidence, lost to follow $\mathrm{up} ; \mathrm{OS}=$ overall survival; PFS = progression-free survival; PR = partial response; $\mathrm{s} . \mathrm{d}$. = standard deviation; $\mathrm{SD}=$ stable disease

also successfully treated patients with perihilar cholangiocarcinoma with minimally invasive ECT treatment. ${ }^{33}$

In our study, we showed that ECT performed best (in terms of progression-free survival and overall survival) in lesions $<6 \mathrm{~cm}$ in diameter compared to lesions $>6 \mathrm{~cm}$ in diameter $(\mathrm{p}=0.0209$ and $p=0.0322$, respectively). These results demonstrate that the technique is effective even in lesions of large size, up to $6 \mathrm{~cm}$, significantly larger than lesions addressable with thermal ablation techniques.

Furthermore, we proved that the effectiveness of ECT is independent of the localization of the lesions: distant, close or adjacent to vital structures, with the latter being devoid of therapeutic options.

We observed that progression-free survival and overall survival in our cohort of patients were similar for all primary histologies considered: colorectal metastases, breast cancer metastases, and hepatocellular cancer $(p=0.8781$ and $p=0.8379$, respectively). This result demonstrates the effectiveness of ECT and bleomycin independent of the histology of the treated lesions.

Apart from its effectiveness, one of the most important advantages of ECT is that it spares colla- genous healthy structures such as vessels and bile ducts. ${ }^{26}$ ECT is repeatable and suitable as a local therapy even if performed between chemotherapy cycles. In conclusion, ECT has the potential to close relevant gaps in the local ablative therapy field: ECT allows the treatment of lesions too large for thermal ablation, nonradiation-sensitive tumors, and lesions adjacent to radiation-vulnerable organs.

The various thermo, radio- and chemoablative procedures as well as endovascular and percutaneous therapies do not compete with each other but complement each other and are used supplementarily in the hands of experienced interventionalists. Radiological-interventional expertise implies that a broad spectrum of procedures and technologies must be mastered. Too many evidence-based recommendations for sequencing minimally invasive procedures do not yet exist. The scarce knowledge is limited to the combination of TACE and RFA in larger HCCs, where thermal ablation alone is limited. ${ }^{40}$ It can be considered an imperative task of the interventional community to generate this same evidence as soon as possible.

In our study, we demonstrated that ECT was well tolerated by the patients, and no serious ad- 
verse events were observed during the procedure or in the follow-up; side effects were limited in number and intensity, and no relevant pain or systemic side effects were observed.

This study has several limitations: a retrospective design, the limited number of cases and their heterogeneity in terms of diagnosis of primary tumor, localization, and previous treatments. This is a first experience conducted in our center and therefore includes all patients treated in the observation period. Moreover, this study well represents the variety of the cohort of patients, which can benefit from the application of ECT in clinical practice.

In one case, where $100 \%$ technical success could not be achieved, a plastic bile duct stent was placed at the margin of the target lesion. The generator reported inadequate discharges at the two electrodes closest to the biliary stent. Although several discharge cycles were performed, residual contrast uptake in this area was documented in the postinterventional MR control after 48 hours. However, it cannot be concluded from this single case observation that bile duct stents per se represent a limitation to ECT.

In addition to these limitations, our study provides further evidence on the effectiveness of ECT in the treatment of liver metastases of different origins, different sizes and locations. This treatment provides long-term local tumor control as well as long progression-free survival (mean progressionfree survival of $9.0 \pm 8.2$ months). ECT, therefore, provides an effective valuable option for the treatment of unresectable liver metastases not amenable to other ablative techniques.

\section{References}

1. Global Burden of Disease Cancer Collaboration; Fitzmaurice $C$, Allen $C$, Barber RM, Barregard L, Bhutta ZA, Brenner $\mathrm{H}$, et al. Global, regional, and national cancer Incidence, mortality, years of life lost, years lived with disability, and disability-adjusted life-years for 32 cancer groups, 1990 to 2015: a systematic analysis for the Global Burden of Disease Study. JAMA Oncol 2017; 3: 524. doi: 10.1001/jamaoncol.2016.5688

2. Valery PC, Laversanne M, Clark PJ, Petrick JL, McGlynn KA, Bray F. Projections of primary liver cancer to 2030 in 30 countries worldwide. Hepatology 2018; 67: 600-11. doi: 10.1002/hep.29498

3. Engstrand J, Nilsson H, Strömberg C, Jonas E, Freedman J. Colorectal cancer liver metastases - a population-based study on incidence, management and survival. BMC Cancer 2018; 18: 78. doi: 10.1186/s12885-017-3925-x

4. HackI C, Neumann P, Gerken M, Loss M, Klinkhammer-Schalke M, Schlitt HJ. Treatment of colorectal liver metastases in Germany: a ten-year populationbased analysis of 5772 cases of primary colorectal adenocarcinoma. BMC Cancer 2014; 14: 810. doi: 10.1186/1471-2407-14-810

5. Van Cutsem E, Cervantes A, Adam R, Sobrero A, Van Krieken JH, Aderka $D$, et al. ESMO consensus guidelines for the management of patients with metastatic colorectal cancer. Ann Oncol 2016; 27: 1386-422. doi: 10.1093/ annonc/mdw235
6. Goldberg SN, Gazelle GS, Mueller PR. Thermal ablation therapy for focal malignancy: a unified approach to underlying principles, techniques, and diagnostic imaging guidance. Am J Roentgenol 2000; 174: 323-31. doi: 10.2214/ajr.174.2.1740323

7. Glass LF, Jaroszeski M, Gilbert R, Reintgen DS, Heller R. Intralesional bleomycin-mediated electrochemotherapy in 20 patients with basal cell carcinoma. J Am Acad Dermatol 1997; 37: 596-9. doi: 10.1016/S0190-9622(97)70178-6

8. Marty M, Sersa G, Garbay JR, Gehl J, Collins CG, Snoj M, et al. Electrochemotherapy - an easy, highly effective and safe treatment of cutaneous and subcutaneous metastases: results of ESOPE (European Standard Operating Procedures of Electrochemotherapy) study. Eur J Cancer Suppl 2006; 4: 3-13. doi: 10.1016/j.ejcsup.2006.08.002

9. Clover AJP, de Terlizzi F, Bertino G, Curatolo P, Odili J, Campana LG, et al. Electrochemotherapy in the treatment of cutaneous malignancy: outcomes and subgroup analysis from the cumulative results from the pan-European International Network for Sharing Practice in Electrochemotherapy database for 2482 lesions in 987 patients (2008-2019). Eur J Cancer 2020; 138: 30-40. doi: 10.1016/j.ejca.2020.06.020

10. Plaschke CC, Bertino G, McCaul JA, Grau JJ, de Bree R, Sersa G, et al. European Research on Electrochemotherapy in Head and Neck Cancer (EURECA) project: results from the treatment of mucosal cancers. Eur $J$ Cancer 2017; 87: 172-81. doi: 10.1016/j.ejca.2017.10.008

11. Bertino G, Sersa G, De Terlizzi F, Occhini A, Plaschke CC, Groselj A, et al. European Research on Electrochemotherapy in Head and Neck Cancer (EURECA) project: results of the treatment of skin cancer. Eur J Cancer 2016; 63: 41-52. doi: 10.1016/j.ejca.2016.05.001

12. Gasljevic G, Edhemovic I, Cemazar M, Brecelj E, Gadzijev EM, Music MM, et al. Histopathological findings in colorectal liver metastases after electrochemotherapy. PLOS ONE 2017; 12: e0180709. doi: 10.1371/journal. pone.0180709

13. Brloznik M, Boc N, Sersa G, Zmuc J, Gasljevic G, Seliskar A, et al. Radiological findings of porcine liver after electrochemotherapy with bleomycin. Radiol Oncol 2019; 53: 415-26. doi: 10.2478/raon-2019-0049

14. Zmuc J, Gasljevic G, Sersa G, Edhemovic I, Boc N, Seliskar A, et al. Large Liver Blood Vessels and Bile Ducts Are Not Damaged by Electrochemotherapy with Bleomycin in Pigs. Sci Rep 2019; 9: 3649. doi: 10.1038/s41598-01940395-y

15. Cornelis FH, Korenbaum C, Ben Ammar M, Tavolaro S, Nouri-Neuville M, Lotz JP. Multimodal image-guided electrochemotherapy of unresectable liver metastasis from renal cell cancer. Diagn Interv Imag 2019; 100: 309-11. doi: 10.1016/j.diii.2019.01.001

16. Edhemovic I, Brecelj E, Cemazar M, Boc N, Trotovsek B, Djokic M, et al. Intraoperative electrochemotherapy of colorectal liver metastases: a prospective phase II study. EJSO 2020; 46: 1628-33. doi: 10.1016/j. ejso.2020.04.037

17. Mali B, Gorjup V, Edhemovic I, Brecelj E, Cemazar M, Sersa G, et al. Electrochemotherapy of colorectal liver metastases - an observational study of its effects on the electrocardiogram. BioMed Eng OnLine 2015; 14(Suppl 3): S5. doi: 10.1186/1475-925X-14-S3-S5

18. Jarm T, Krmac T, Magjarevic R, Kos B, Cindric H, Miklavcic D. Investigation of safety for electrochemotherapy and irreversible electroporation ablation therapies in patients with cardiac pacemakers. BioMed Eng OnLine 2020; 19: 85. doi: 10.1186/s12938-020-00827-7

19. Boc N, Edhemovic I, Kos B, Marolt Music M, Brecelj E, Trotovsek B, et al. Ultrasonographic changes in the liver tumors as indicators of adequate tumor coverage with electric field for effective electrochemotherapy. Radiol Oncol 2018; 52: 383-91. doi: 10.2478/raon-2018-0041

20. Bertacchini C. Cliniporator: medical electroporation of tumors. In: Miklavcic D, editor. Handbook of Electroporation. Cham: Springer International Publishing; 2017. p. 1-36.

21. Gehl J, Sersa G, Matthiessen LW, Muir T, Soden D, Occhini A, et al. Updated standard operating procedures for electrochemotherapy of cutaneous tumours and skin metastases. Acta Oncol 2018; 57: 874-82. doi: 10.1080/0284186X.2018.1454602

22. Ruers T, Van Coevorden F, Punt CJ, Pierie JE, Borel-Rinkes I, Ledermann JA, et al. Local treatment of unresectable colorectal liver metastases: results of a randomized phase ii trial. J Nat/ Cancer / 2017; 109: djx015. doi: 10.1093/ jnci/djx015 
23. Slovak R, Ludwig JM, Gettinger SN, Herbst RS, Kim HS. Immuno-thermal ablations - boosting the anticancer immune response. J Immunother Cancer 2017; 5: 78. doi: 10.1186/s40425-017-0284-8

24. Camacho JC, Petre EN, Sofocleous CT. Thermal ablation of metastatic colon cancer to the liver. Semin Intervent Radiol 2019; 36: 310-8. doi: 10.1055/s0039-1698754

25. Olson R, Mathews L, Liu M, Schellenberg D, Mou B, Berrang T, et al. Stereotactic ablative radiotherapy for the comprehensive treatment of 1-3 Oligometastatic tumors (SABR-COMET-3): study protocol for a randomized phase III trial. BMC Cancer 2020; 20: 380. doi: 10.1186/s12885-020-06876-4

26. Kovács A, Bischoff P, Haddad H, Kovács G, Schaefer A, Zhou W, et al. Personalized image-guided therapies for local malignencies: interdisciplinary options for interventional radiology and interventional radiotherapy. Front Oncol 2021; 11: 616058. doi: 10.3389/fonc.2021.616058

27. Campanacci L, Bianchi G, Cevolani L, Errani C, Ciani G, Facchini G, et al. Operating procedures for electrochemotherapy in bone metastases: results from a multicenter prospective study on 102 patients. Eur J Surg Oncol 2021; 47: 2609-17. doi: 10.1016/j.ejso.2021.05.004

28. Edhemovic I, Gadzijev EM, Brecelj E, Miklavcic D, Kos B, Zupanic A, et al. Electrochemotherapy: a new technological approach in treatment of metastases in the liver. Technol Cancer Res Treat 2011; 10: 475-85. doi: 10.7785/ tcrt.2012.500224

29. Edhemovic I, Brecelj E, Gasljevic G, Marolt Music M, Gorjup V, Mali B, et al. Intraoperative electrochemotherapy of colorectal liver metastases. J Surg Oncol 2014; 110: 320-7. doi: 10.1002/jso.23625

30. Coletti L, Battaglia V, De Simone P, Turturici L, Bartolozzi C, Filipponi F. Safety and feasibility of electrochemotherapy in patients with unresectable colorectal liver metastases: a pilot study. Int J Surg 2017; 44: 26-32. doi: 10.1016/j.ijsu.2017.06.033

31. Djokic M, Cemazar M, Popovic P, Kos B, Dezman R, Bosnjak M, et al. Electrochemotherapy as treatment option for hepatocellular carcinoma, a prospective pilot study. EJSO 2018; 44: 651-7. doi: 10.1016/j. ejso.2018.01.090

32. Djokic M, Dezman R, Cemazar M, Stabuc M, Petric M, Smid LM, et al Percutaneous image guided electrochemotherapy of hepatocellular carcinoma: technological advancement. Radiol Oncol 2020; 54: 347-52. doi: 10.2478/raon-2020-0038

33. Tarantino L, Busto G, Nasto A, Nasto RA, Tarantino P, Fristachi R, et al. Electrochemotherapy of cholangiocellular carcinoma at hepatic hilum: a feasibility study. EJSO 2018; 44: 1603-9. doi: 10.1016/j.ejso.2018.06.025

34. Granata V, Palaia R, Albino V, Piccirillo M, Venanzio Setola S, Petrillo A, et al. Electrochemotherapy of cholangiocellular carcinoma at hepatic hilum: a case report. Eur Rev Med Pharmacol Sci 2020; 24: 7051-7. doi: 10.26355/ eurrev_202006_21698

35. Corrado G, Cutillo G, Fragomeni SM, Bruno V, Tagliaferri L, Mancini E, et al. Palliative electrochemotherapy in primary or recurrent vulvar cancer. Int $J$ Gynecol Cancer 2020; 30: 927-31. doi: 10.1136/ijgc-2019-001178

36. Perrone AM, Galuppi A, Pirovano C, Borghese G, Covarelli P, De Terlizzi F, et al. Palliative Electrochemotherapy in vulvar carcinoma: preliminary results of the ELECHTRA (Electrochemotherapy Vulvar Cancer) multicenter study. Cancers 2019; 11: 657. doi: 10.3390/cancers11050657

37. Perrone AM, Ferioli M, Argnani L, De Terlizzi F, Pirovano $C$, Covarelli $P$, et al. Quality of life with vulvar carcinoma treated with palliative electrochemotherapy: The ELECHTRA (ELEctroCHemoTherapy vulvaR cAncer) Study. Cancers 2021; 13: 1622 . doi: 10.3390/cancers 13071622

38. Andresciani F, Faiella E, Altomare C, Pacella G, Beomonte Zobel B, Grasso RF. Reversible electrochemotherapy (ECT) as a treatment option for local RCC recurrence in solitary kidney. Cardiovasc Intervent Radiol 2020; 43: 1091-4. doi: $10.1007 / \mathrm{s} 00270-020-02498-2$

39. Tarantino L, Busto G, Nasto A, Fristachi R, Cacace L, Talamo M, et al. Percutaneous electrochemotherapy in the treatment of portal vein tumo thrombosis at hepatic hilum in patients with hepatocellular carcinoma in cirrhosis: A feasibility study. WJG 2017; 23: 906. doi: 10.3748/wjg.v23.i5.906

40. Liu F, Chen M, Mei J, Xu L, Guo R, Lin X, et al. Transarterial chemoembolization combined with radiofrequency ablation in the treatment of stage B1 intermediate hepatocellular carcinoma. J Oncol 2019; 2019: 1-7. doi: $10.1155 / 2019 / 6298502$ 